\title{
Dietary modulation of gluten sensitivity in a naturally occurring enteropathy of Irish setter dogs
}

\author{
E J Hall, R M Batt
}

\begin{abstract}
Gluten sensitivity in a naturally occurring enteropathy of Irish setter dogs, and the effects. of excluding dietary cereal from birth on the subsequent response to gluten challenge were investigated. Peroral jejunal biopsy specimens were obtained at 1 year of age for morphometric and biochemical examinations, and intestinal permeability was assessed using ${ }^{51} \mathrm{Cr}$-ethylenediaminetetraacetic acid. Affected setters, reared on a normal wheat containing diet, exhibited partial villus atrophy, intraepithelial lymphocyte infiltration, reduced brush border alkaline phosphatase activity, and increased intestinal permeability. Gluten sensitivity was shown by introduction of a gluten free diet, which resulted in resolution of morphological and biochemical abnormalities and decreased intestinal permeability, and subsequent gluten challenge, which resulted in relapse. In contrast, littermates reared exclusively on a cereal free diet showed minimal changes when challenged with gluten, apart from intraepithelial lymphocyte infiltration. These findings document a gluten sensitive enteropathy in Irish setters and indicate that exclusion of dietary cereal from birth may modify subsequent expression of the disease.
\end{abstract}

The mechanism by which gluten damages the intestinal mucosa in coeliac disease (gluten sensitive enteropathy) and the influence that genetic and environmental factors have on the expression of the disease remain unclear. ${ }^{12}$ Investigations in patients with coeliac disease are complicated by various factors. Sequential investigations of patients before and during the development of the disease are neither practical nor ethical, and accurate diagnosis may require multiple intestinal biopsy.' Studies of histocompatibility antigens suggest a complex genetic influence on the expression of the disease, ${ }^{3}$ and discordant findings in some identical twins ${ }^{4}$ and HLA-DR identical siblings ${ }^{5}$ emphasise the potential influence of environmental factors. In addition, primary abnormalities in treated patients may be obscured by inadvertent gluten ingestion or any long term effects of severe mucosal damage. ${ }^{2}$ Examination of a suitable animal model might help elucidate the mechanisms involved.

The incidence of coeliac disease seems to be declining in the UK and Ireland..$^{6-8}$ It has been speculated that changes in environmental factors, such as feeding practices, may be responsible for the decline, but controlled prospective studies are lacking. Complementary studies on an appropriate animal model could again prove useful. Experimentally, gluten sensitivity has been established in rats by administration of triparanol, ${ }^{9}$ or by feeding a niacin deficient diet $^{10}$ or the mannosidase inhibitor, swainsonine, ${ }^{11}$ and the mechanisms of mucosal damage have been explored in models such as graft $v$ host disease. ${ }^{12}$ These models, however, do not permit investigation of the influence of environmental factors on the expression of disease, and an animal model of a naturally occurring gluten sensitive enteropathy would be of value.

There is anecdotal evidence of gluten sensitivity in animals ${ }^{13}$ and a single case report in a cynomolgus macaque, ${ }^{1+}$ but no reliable, naturally occurring animal model has yet been established. However, a naturally occurring enteropathy in Irish setters, characterised by partial villus atrophy, intraepithelial lymphocyte infiltration, and specific biochemical abnormalities has been reported. ${ }^{15}{ }^{16}$ Clinical signs of poor appetite, weight loss or poor weight gain, and chronic intermittent diarrhoea were seen, and the enteropathy was subsequently shown to be wheat sensitive. ${ }^{17}$ Recovery of alkaline phosphatase activity and an increase in villus height were documented when animals were fed a wheat free diet; relapse on subsequent challenge with wheat flour resulted in partial villus atrophy and a selective loss of brush border alkaline phosphatase activity. The damaging component of wheat flour was not identified, but was suspected to be gluten.

Sequential studies of dogs bred from affected setters and reared on a normal wheat containing diet showed that the development of partial villus atrophy, intraepithelial lymphocyte infiltration, and biochemical changes occurred only if cereal was present in the diet. ${ }^{18}{ }^{19}$ Furthermore, the characteristic loss of brush border alkaline phosphatase and aminopeptidase $\mathrm{N}$ activities in this enteropathy, although secondary to the presence dietary cereal, was a specific effect, as disaccharidases were not affected. Littermates reared exclusively on a cereal free diet showed no morphological or biochemical evidence of intestinal damage. Both groups of littermates, however, exhibited increased permeability to ${ }^{51} \mathrm{Cr}$-ethylenediaminetetra-acetic acid (EDTA), suggesting that an underlying permeability defect might be involved in the pathogenesis of the condition. ${ }^{20}$ Although this disease cannot be considered completely analagous to coeliac disease, it may be a useful model for the investigation of the mechanism of intestinal damage by dietary cereal.

One aim of the present study was to determine whether gluten is the toxic factor in wheat responsible for the mucosal abnormalities in wheat sensitive enteropathy of Irish setters. The
Department of Veterinary E J Hall

Correspondence to: DrE J Hall, Department of Veterinary Pathology, University of Liverpool, $\mathrm{PO}$ Box 147, Liverpool L69 3BX. Accepted for publication 14 May 1991 
response of affected dogs reared on a normal diet to gluten withdrawal and subsequent oral challenge with gluten was monitored by assessment of intestinal morphology, biochemistry, and permeability. The second aim of the study was to determine if lack of exposure to gluten early in life influences the subsequent response to challenge, by investigation of the effect of gluten challenge in affected progeny that had been reared exclusively on a cereal free diet.

\section{Methods}

\section{GROUPS OF ANIMALS}

Eight Irish setter dogs, progeny of Irish setters with documented wheat sensitive enteropathy, were studied. The dogs were part of a sequential study examining the development of wheat sensitive enteropathy reported elsewhere. ${ }^{18} 19$ They were allotted to two equal groups at 5-6 weeks of age and weaned. One group of dogs was reared exclusively on a cereal free diet of rabbit and turkey meat (Mr Dog, Pedigree Petfoods). The other group of littermates was reared on a normal diet consisting of rabbit and turkey meat and also cereals containing wheat (Pedigree Chum Mixer, Pedigree Petfoods). Control dogs were seven age matched clinically healthy Irish setters fed the same diet of meat and cereal as the normal diet group.

Investigations were performed from 12 months of age, by which time the normal diet group had developed wheat sensitive enteropathy. ${ }^{18} 19$ The normal diet group was then studied in remission after six weeks on a gluten free diet. Gluten challenge studies were subsequently performed on both groups using $1 \mathrm{~g}$ of gluten (Sigma Chemical Co Ltd) added to the food each day for six weeks.

\section{MORPHOLOGICAL AND BIOCHEMICAL STUDIES}

Peroral jejunal biopsy specimens were obtained for morphological and biochemical studies as described previously. ${ }^{21}$ Formalin fixed specimens were processed routinely for the measurement of villus height and intraepithelial lymphocyte density. Fresh tissue was subjected to analytical subcellular fractionation on reorientating sucrose density gradients and the specific activities and gradient distributions of organelle marker enzymes were determined.

\section{INTESTINAL PERMEABILITY}

Permeability to ${ }^{51} \mathrm{Cr}$-EDTA was assessed in each dog one week before jejunal biopsy using the procedure validated for dogs. ${ }^{22}$ Results were expressed as the 24 hour percentage urinary recovery of the orally administered dose of

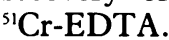

\section{STATISTICAL ANALYSIS}

Results are expressed as mean (SEM). After testing for homogeneity of variance by Levene's test, statistical comparisons between normal diet, cereal free diet, and control groups were by analysis of variance and Newman-Keul's test.
Comparisons between different diets within the same group were made by Student's paired $t$ test. Differences with a $p$ value $<0.05$ were considered significant.

\section{Results}

When the dogs reared on a normal diet were put on a gluten free diet they showed noticeable clinical improvement; their diarrhoea stopped and their body weight (kg, mean (SEM)) increased in six weeks from $17 \cdot 9(1 \cdot 2)$ to $21(1 \cdot 0)$ $(p<0.05)$. Gluten challenge at six weeks resulted in a recurrence of diarrhoea and a reduction in body weight to $18 \cdot 1(0.9)(p<0 \cdot 05)$. However, when the dogs in the cereal free diet group were challenged with gluten in their diet for six weeks mild diarrhoea only occurred and their weight loss, from 19.7 (1.2) to $18.9(0.4)(p>0.05)$, was not significant. No valid comparisons of weights between the two groups could be made because of the sexual dimorphism exhibited in this breed and the different numbers of dogs and bitches in the groups.

\section{MORPHOLOGICAL STUDIES}

In the normal diet group, villus height increased on the gluten free diet and was no longer significantly less than in the controls; subsequent gluten challenge produced a significant reduction in villus height (Fig 1A). In the cereal fed group, gluten challenge produced a small reduction in villus height which was not significant, although villus height was now significantly lower than in the controls. The number of intraepithelial lymphocytes in the normal diet group decreased to control values on a gluten free diet, and returned to the high initial values with gluten challenge. The number of intraepithelial lymphocyte also increased significantly in the cereal fed diet group with gluten challenge (Fig 1B).

\section{ENZYME SPECIFIC ACTIVITIES}

The activity of alkaline phosphatase in homogenates from the normal diet group showed a noticeable increase on a gluten free diet and a reduction with gluten challenge, although at all times activities were less than in the control dogs (Fig 1C). In the cereal free diet group, gluten challenge produced a small decrease in alkaline phosphatase activity that was not significant, although activity was now significantly lower than in the controls.

The activity of aminopeptidase $\mathrm{N}$ did not alter significantly in the normal diet group after dietary manipulation and remained less than in the controls, but there was a significant fall in activity in the cereal free diet group with gluten challenge (Fig 1D).

Feeding the gluten free diet to the normal diet group produced a considerable increase in $\gamma$-glutamyl transferase activity from a mean value less than that of the control dogs to a value greater than that of the controls, and subsequent gluten challenge reduced the activity to the control value (Fig 1E). Gluten challenge had no effect on the activity of $\gamma$-glutamyl transferase in the cereal free diet group. 
In the normal diet group, the specific activity of $\mathrm{Zn}$ resistant $\alpha$-glucosidase became significantly less than controls after introduction of the gluten free diet, and the downward trend continued after gluten challenge (Fig $1 F$ ). In the cereal free diet group, the activity of $\mathrm{Zn}$ resistant $\alpha$-glucosidase was initially less than in controls and was not affected by gluten challenge.

ANALYTICAL SUBCELLULAR FRACTIONATION

In the normal diet group, six weeks of feeding a gluten free diet produced a noticeable recovery of the particulate and soluble activities of alkaline phosphatase; subsequent gluten challenge caused a loss of these activities (Fig 2). In the cereal free diet group, gluten challenge
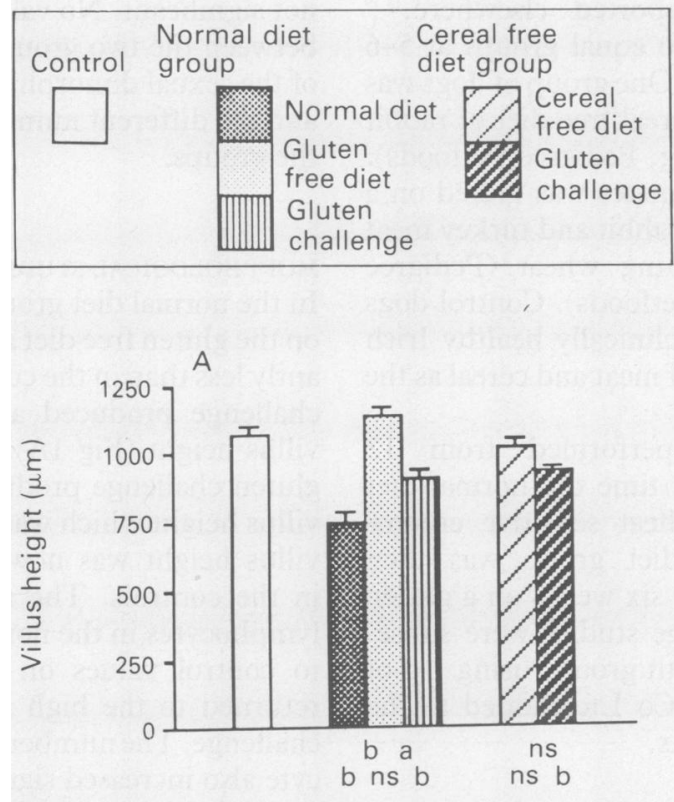

Figure 1: Effects of dietary gluten on jejunal villus height $(A)$, intraepithelial lymphocyte (IEL) density $(B)$, and activities of alkaline phosphatase $(C)$, aminopeptidase $N(D), \gamma$ glutamyl transferase $(\gamma G T)$ $(E)$, and $Z n$ resistant $\alpha$ glucosidase $(F)$. Results are from affected Irish setters reared on a wheat containing diet, after 6 weeks on a gluten free diet and after 6 weeks' gluten challenge, and from littermates reared on a cereal free diet before and after 6 weeks' gluten challenge. Letters designate statistical comparisons between treatments (above) and between affected dogs and controls (below) as follows: $n s=$ not significant, $a=p<0.05, b=p<0.01$.
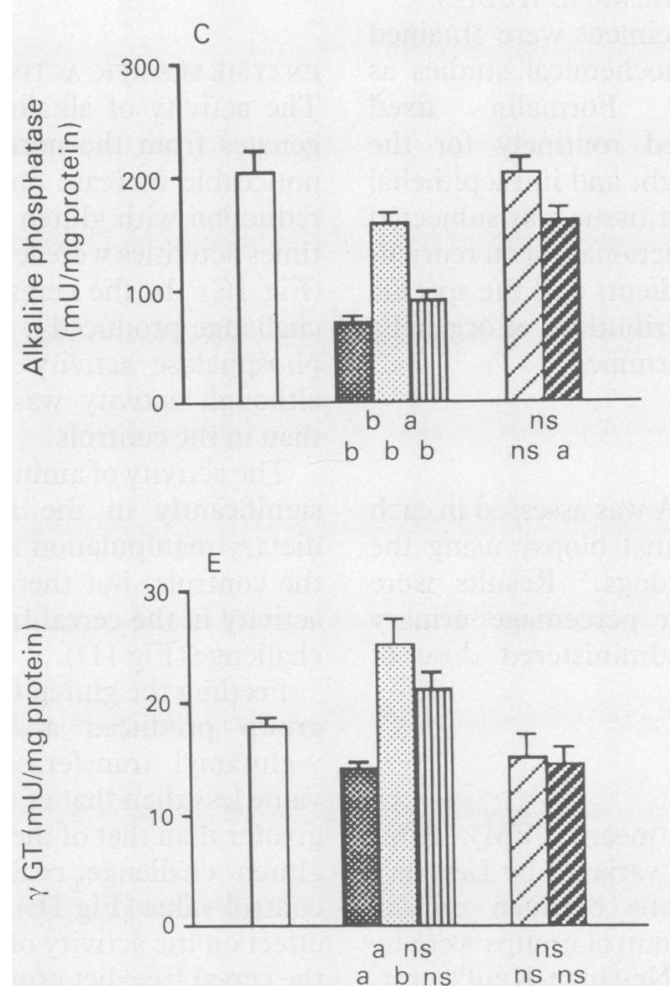

resulted in a loss of some of the particulate brush border component of alkaline phosphatase.

After introduction of the gluten free diet to the normal diet group, aminopeptidase $\mathrm{N}$ activity showed a more defined particulate peak, with a modal density of $1.21 \mathrm{~g} / \mathrm{ml}$ comparable with that of controls, although there was little change in the overall relative frequency; gluten challenge returned the distribution to a wider peak with a lighter modal density (Fig 3). In the cereal free diet group, gluten challenge resulted in a loss of some of the particulate brush border component of aminopeptidase $\mathrm{N}$.

Initially, the gradient distribution of $\gamma$ glutamyl transferase activity in the normal diet group had a reduced particulate peak with a lighter modal density compared with the distribution in the controls (Fig 4). After the gluten free diet, the distribution exhibited a distinct brush border peak, with a normal modal density of $1.21 \mathrm{~g} / \mathrm{ml}$. The size of this peak decreased to control values with gluten challenge, but there was no shift in modal density. The gradient distribution of $\gamma$-glutamyl transferase in the cereal free diet group was largely unaltered by gluten challenge. The modal density of the particulate peak of $\mathrm{Zn}$ resistant $\alpha$-glucosidase
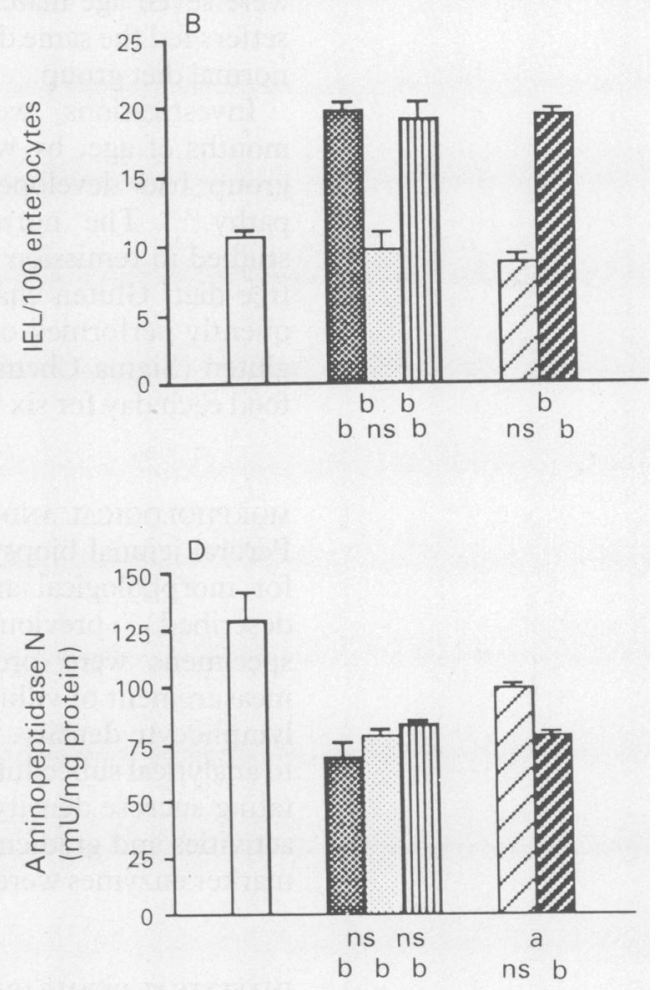
was identical to the controls in both groups of $\operatorname{dogs}^{19}$ and remained unchanged with dietary manipulation (not shown).

\section{INTESTINAL PERMEABILITY}

The permeability to ${ }^{51} \mathrm{Cr}$-EDTA in the normal diet group decreased with the introduction of the gluten free diet, but only to the same level as in the cereal free diet group, which was greater than in the controls. Permeability returned to initial high values in the normal diet group after gluten challenge. In the cereal free group there was no change in permeability to ${ }^{51} \mathrm{Cr}$-EDTA with gluten challenge, although at all times permeability was greater than in the controls (Fig 5).

\section{Discussion}

The initial diagnosis of coeliac disease depends on verification of the recovery of an abnormal intestinal mucosa on a gluten free diet, ${ }^{23}$ but clinical improvement, ${ }^{24}$ intestinal enzyme activities, ${ }^{25}$ and intestinal permeability ${ }^{26}$ may take several months to return to normal after gluten withdrawal, even if deliberate or inadvertant gluten ingestion does not occur. In addition, evidence of relapse on subsequent gluten challenge may be crucial for the accurate diagnosis of coeliac disease in order to distinguish this dis- order from a transient gluten intolerance. ${ }^{27} 28$ However, the length of time of exposure of the intestinal mucosa to gluten before abnormalities recur has not yet been established, ${ }^{2429}$ and in children may actually be several years. ${ }^{28}$ Confusion has also arisen because the amount of gluten, or gluten equivalent, used to challenge coeliac patients has varied considerably both with regard to the total amount administered and whether it was given as single or repeated doses. ${ }^{29}$ It has been suggested that the age, immunological status, endocrinological maturity, and genetic make-up of patients, and the size of gluten challenge all influence the response to gluten. ${ }^{29}$ An appropriate animal model would be of value in examining the mechanism by which gluten can damage the intestinal mucosa.

Our study clearly shows that the enteropathy in Irish setters is responsive to the presence of gluten in the diet. Considerable clinical improvement, recovery from jejunal morphological and biochemical abnormalities, and reduction of intestinal permeability were seen in affected progeny reared on a normal diet within six weeks of starting a gluten free diet; relapse occurred when these dogs were challenged with 1 g gluten per day for six weeks. The dose of gluten given to the dogs was relatively small, equivalent to approximately $15 \mathrm{~g}$ of wheat flour or one thick slice of white bread $(25 \mathrm{~g})$ per day, ${ }^{29}$ and was
Figure 2: Subcellular distribution of alkaline phosphatase activity after isopycnic centrifugation of postnuclear supernatants from jejunal biopsy specimens on reorientating sucrose density gradients. Homogenates were from affected dogs reared on a wheat containing diet $(N D)$ $(A)$, after 6 weeks on a gluten free diet $(G F D)(B)$, and after 6 weeks' gluten challenge $(G C)(C)$, and from littermates reared on a cereal free diet $(C F D)$ before $(D)$ and after (E) 6 weeks' GC. Graphs show relative frequency-density histograms; the areas comparing affected (stippled, mean (SEM)) with control animals $(C)$ (solid line) are proportional to their specific activity ( $m U$ ) $m g$ protein). For the affected dogs reared on a ND, the mean percentage recoveries for each enzyme (ND, GFD, GC, C respectively), with their relative specific activities in parenthesis (ND:C, GFD:C, GC:C respectively) were 94,95 $93,90(0.34,0.77,0.44)$ For their littermates reared on a CFD, the mean percentage recoveries for each enzyme (CFD, GC respectively), with their relative specific activities in parenthesis (CFD:C, GC:C respectively) were 91,105 $(0 \cdot 99,0 \cdot 78)$.
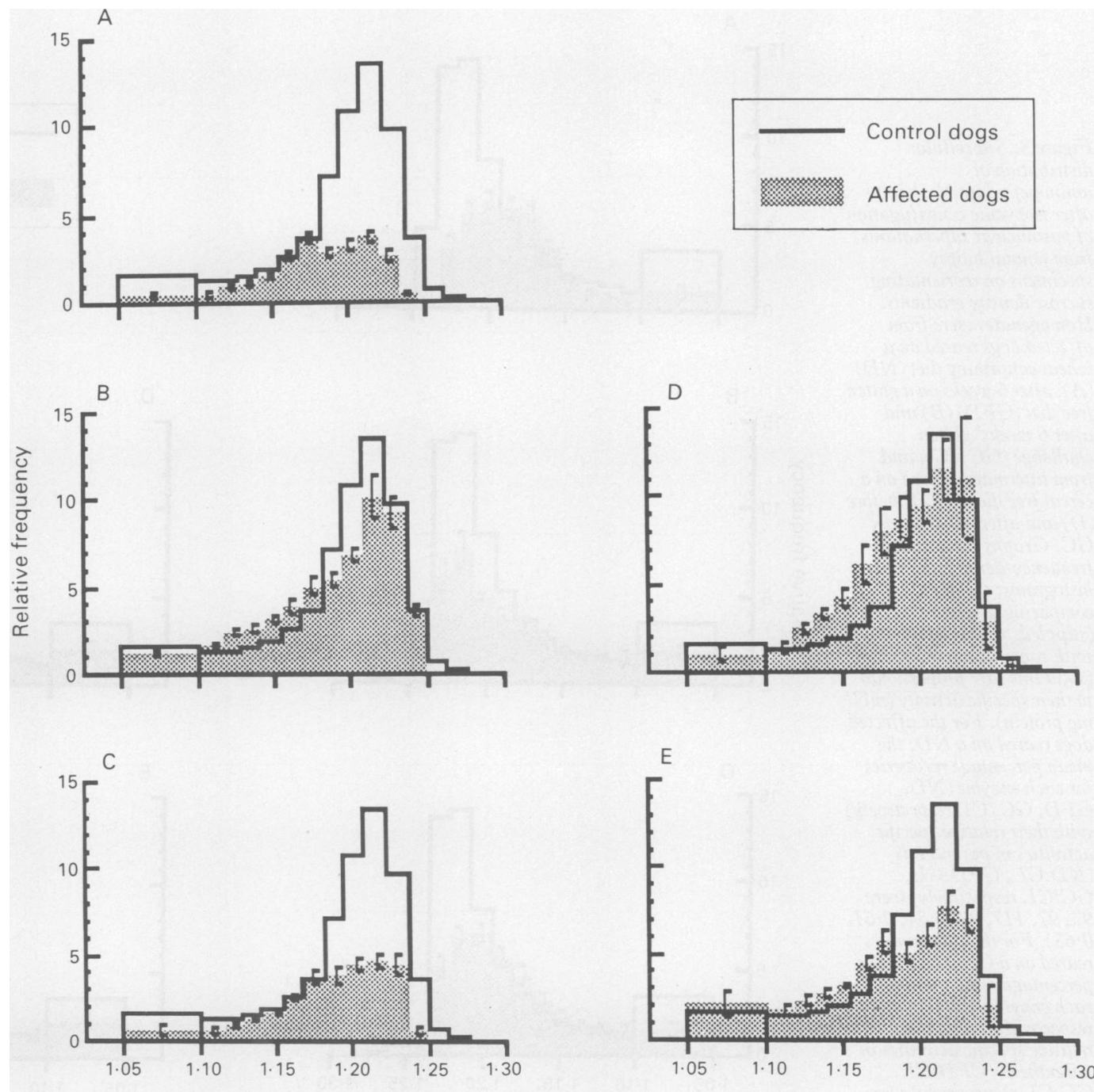
chosen as a suitable physiological amount. However, gluten challenge had much less profound effects on littermates reared exclusively on a cereal free diet. This study, therefore, not only defines the first naturally occurring animal model of gluten sensitivity, but also supports the hypothesis that lack of exposure to gluten in early life may modify the subsequent response to gluten challenge in genetically susceptible animals.

In the normal diet group, cessation of diarrhoea and weight gain on introduction of the gluten free diet were associated with an increase in villus height to normal, while subsequent gluten challenge resulted in clinical relapse and partial villus atrophy. Photomicrographs showing the characteristic appearance of biopsy specimens in affected setters on wheat containing and cereal free diets have been published previously. ${ }^{17}{ }^{18}$ Unfortunately, the presence of any compensatory changes in crypt morphology - for example, crypt hypertrophy during villus atrophy - could not be assessed for two reasons. Firstly, the suction biopsy technique often failed to obtain a full mucosal thickness biopsy specimen reaching the muscularis as canine villi are almost twice the length of human villi. ${ }^{18}$ Secondly, in atrophied specimens the suction technique tended to produce artefactual crypt eversion.
The density of intraepithelial lymphocytes decreased to control values on the gluten free diet, and increased with gluten challenge. In contrast, in the cereal free diet group, there was only mild clinical deterioration and minimal changes in villus height, although intraepithelial lymphocyte density did increase with gluten challenge. Changes in the cellular infiltration of the small intestinal mucosa in coeliac disease in response to gluten challenge have been accepted as evidence of gluten sensitivity, ${ }^{29}$ and a rise in intraepithelial lymphocyte density is a well documented effect of gluten challenge in coeliac patients. ${ }^{30}$ The comparable increases in intraepithelial lymphocyte density in response to gluten in either group of dogs indicates some form of immunological response to gluten irrespective of exposure in early life. However, the differences between the groups in their responses to gluten challenge, assessed by other morphological, biochemical, and permeability measurements, indicate that lack of early exposure to gluten may modify the extent or the rate of the pathological response in genetically susceptible dogs, and that intraepithelial lymphocyte infiltration may not necessarily play a role in the production of intestinal damage.

The presence of gluten in the diet had effects of differing magnitude on the biochemical changes in the normal and cereal free diet
Figure 3: Subcellular distribution of aminopeptidase $N$ activity after isopycnic centrifugation of postnuclear supernatants from jejunal biopsy specimens on reorientating sucrose density gradients. Homogenates were from affected dogs reared on a wheat containing diet $(N D)$ $(A)$, after 6 weeks on a gluten free diet $(G F D)(B)$ and after 6 weeks' gluten challenge $(G C)(C)$, and from littermates reared on a cereal free diet (CFD) before (D) and after $(E) 6$ weeks' GC. Graphs show relative frequency-density histograms; the areas comparing affected (stippled, mean (SEM)) with control animals $(C L)$ (solid line) are proportional to their specific activity ( $m U$ / $m g$ protein). For the affected dogs reared on a ND, the mean percentage recoveries for each enzyme (ND, $G F D, G C, C L$ respectively) with their relative specific activities in parenthesis (ND:CL, GFD:CL, GC:CL respectively) were $97,97,117,90(0.54,0.61$, $0.65)$. For their littermates reared on a $C F D$, the mean percentage recoveries for each enzyme ( $C F D, G C$ respectively), with their relative specific activities in parenthesis (CFD:CL $G C: C L$ respectively) were $88,103(0 \cdot 77,0 \cdot 60)$.
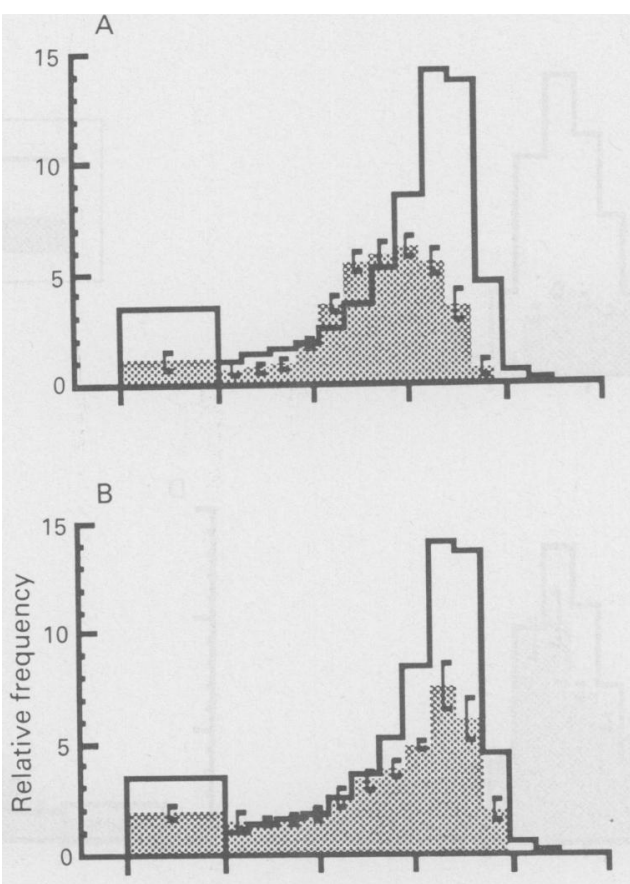

D

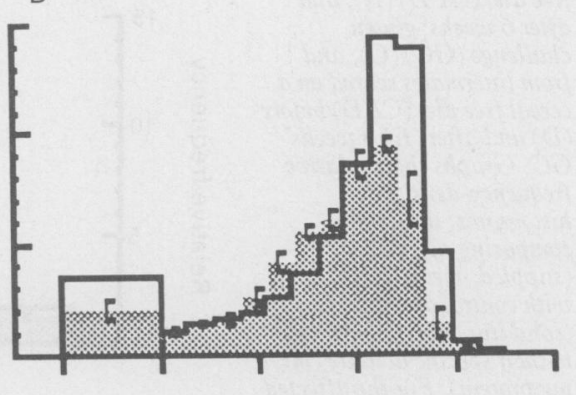

E

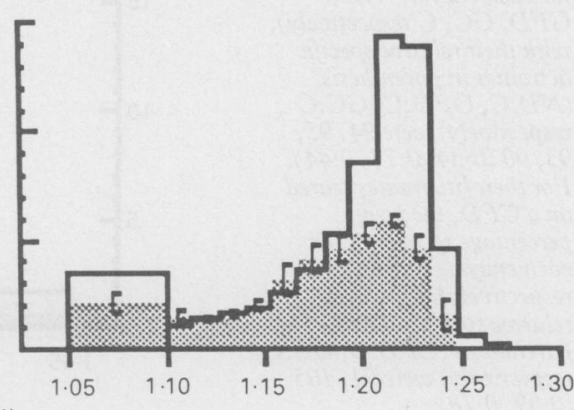


groups. In the normal diet group, there was some recovery of the brush border component of alkaline phosphatase after introduction of a gluten free diet, while subsequent gluten challenge resulted in a noticeable and selective loss of this component of alkaline phosphatase activity. These changes closely resembled those reported previously after introduction of a wheat free diet and subsequent challenge with wheat flour. ${ }^{17}$ However, in the present study, total alkaline phosphatase activity never returned completely to normal. Gluten challenge reduced alkaline phosphatase activity slightly in the cereal free diet group, and there was some selective loss of the brush border component of alkaline phosphatase activity, although this loss was less noticeable than in the normal diet group.

The specific activity of aminopeptidase $\mathrm{N}$ did not change in the normal diet group in response to gluten withdrawal, and remained less than in the controls. However, subcellular fractionation showed some recovery in the brush border peak, as the modal density of the particulate fraction increased and became comparable with that of the controls. Six weeks on a gluten free diet may have been insufficient time for appreciable recovery of aminopeptidase $\mathrm{N}$ activity to occur, since complete recovery of alkaline phosphatase activity did not occur either. Total recovery of these activities in affected Irish setters was

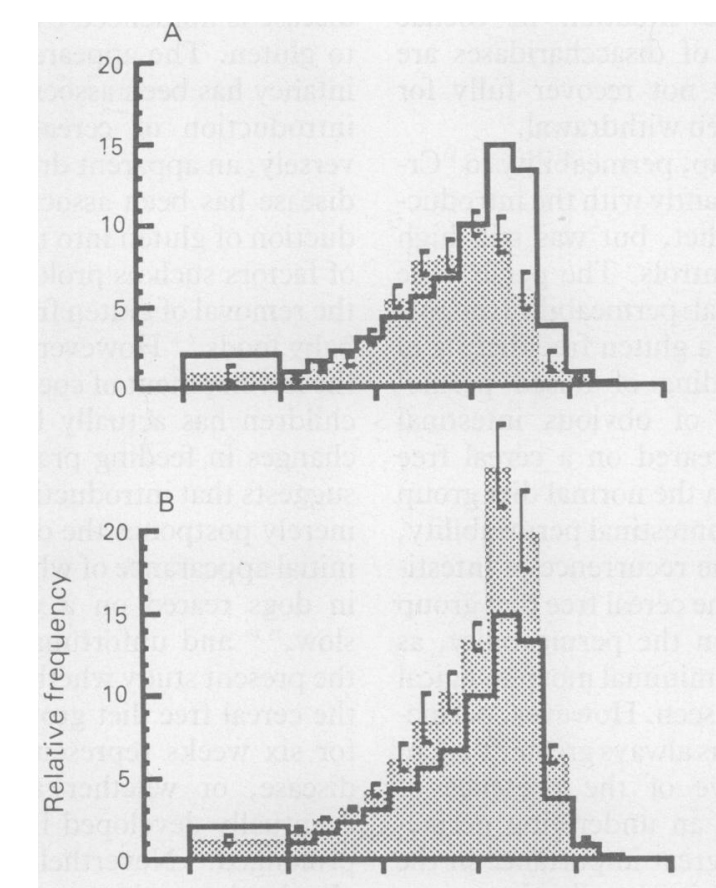

previously reported after five months on a cereal free diet. ${ }^{17}$ and slow recovery of brush border enzymes has been reported in coeliac patients on a gluten free diet ${ }^{25}$ Subsequent gluten challenge did not further reduce the already diminished aminopeptidase $\mathrm{N}$ specific activity in the normal diet group, but subcellular fractionation showed a selective loss of the brush border peak. In the cereal free diet group, aminopeptidase $\mathrm{N}$ activity fell slightly with gluten challenge, and again the brush border component was affected.

The specific activity of $\gamma$-glutamyl transferase increased dramatically, reflecting recovery of the brush border component, when the gluten free diet was introduced to the normal diet group, yet gluten challenge produced only a small reduction in activity in this group and no changes in the cereal free diet group. These findings are consistent with the late appearance, at 12 months of age, of quite moderate reductions in $\gamma$-glutamyl transferase activity in the normal diet group. ${ }^{19}$ It is both alkaline phosphatase and aminopeptidase $\mathrm{N}$ that are most susceptible to damage by gluten in affected Irish setters; relative sparing of $\gamma$-glutamyl transferase activity has been reported both in affected Irish setters ${ }^{15}$ and in coeliac disease. ${ }^{31}$

The gradient distributions of $\mathrm{Zn}$ resistant $\alpha$-glucosidase in both groups were unaffected by the presence of gluten in the diet. This enzyme
Figure 4: Subcellular distribution of $\gamma$-glutamyl transferase activity after isopycnic centrifugation of postnuclear supernatants from jejunal biopsy specimens on reorientating sucrose density gradients.

Homogenates were from affected dogs reared on $a$ wheat containing diet $(N D)$ $(A)$, after 6 weeks on a gluten free diet $(G F D)(B)$, and after 6 weeks' gluten challenge $(G C)(C)$, and from littermates reared on $a$ cereal free diet before $(C F D)$ (D) and after (E) 6 weeks' GC. Graphs show relative frequency-density

histograms; the areas comparing affected

(stippled, mean (SEM))

with control animals (CL)

(solid line) are proportional

to their specific activity $(m U$

mg protein). For the affected

dogs reared on a ND, the

mean percentage recoveries

for each enzyme

(ND, GFD, GC, CL

respectively), with their

relative specific activities

in parenthesis $(N D: C L$,

$G F D: C L, G C: C L$

respectively) were 101,111 ,

$121,113(0.79,1 \cdot 4,1 \cdot 18)$.

For their littermates reared

on a $C F D$, the mean

percentage recoveries for

each enzyme (CFD, GC

respectively), with their

relative specific activities in

parenthesis (CFD:CL,

$G C: C L$ respectively) were

$87,112(0 \cdot 84,0 \cdot 81)$.
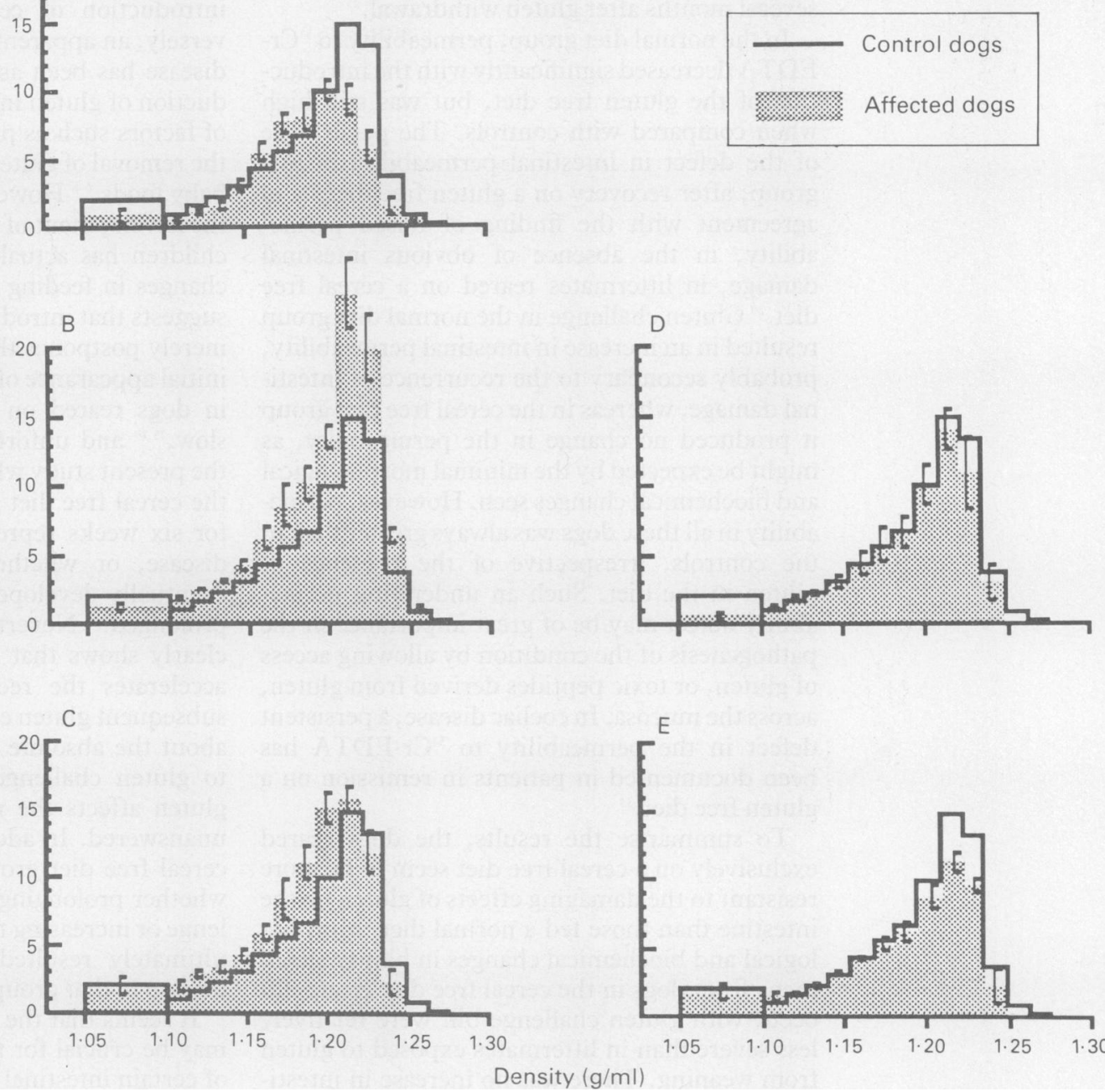

Density ( $\mathrm{g} / \mathrm{ml})$ 
Figure 5: Effects of dietary gluten on intestinal permeability to

${ }^{51} C r-E D T A$. Results are from affected Irish setters reared on a

wheat containing diet, after 6 weeks on a gluten free diet and after 6 weeks' gluten challenge, and from littermates reared on a cereal free diet before and after 6 weeks' gluten challenge.

Letters designate statistical comparisons between treatments

(above) and between affected dogs and controls (below) as

follows: $N S=$ not significant, $a=p<0.05, b=$ $p<0.01$.

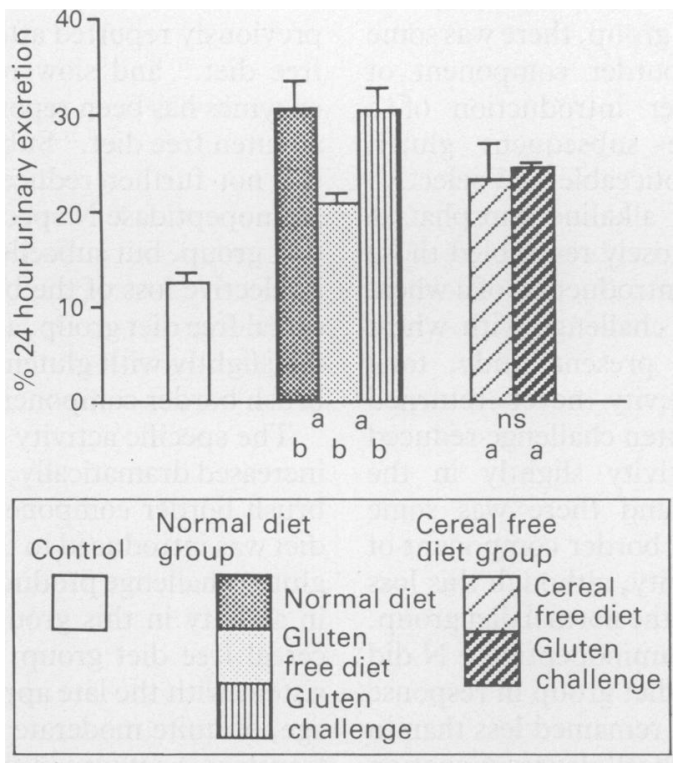

activity, measured using an artificial substrate, is believed to reflect maltase activity, ${ }^{32}$ and the reduced specific activity on a cereal free diet probably reflected lack of induced activity because of the lower carbohydrate content of the diet. ${ }^{19}$ Compared with alkaline phosphatase and aminopeptidase $\mathrm{N}$, the disaccharidases seem relatively resistant to mucosal damage in wheat sensitive enteropathy. ${ }^{19}$ This finding is in marked contrast to the situation in coeliac disease where activities of disaccharidases are invariably low and may not recover fully for several months after gluten withdrawal. ${ }^{25}$

In the normal diet group, permeability to ${ }^{51} \mathrm{Cr}$ EDTA decreased significantly with the introduction of the gluten free diet, but was still high when compared with controls. The persistence of the defect in intestinal permeability in this group, after recovery on a gluten free diet, is in agreement with the finding of raised permeability, in the absence of obvious intestinal damage, in littermates reared on a cereal free diet. ${ }^{20}$ Gluten challenge in the normal diet group resulted in an increase in intestinal permeability, probably secondary to the recurrence of intestinal damage, whereas in the cereal free diet group it produced no change in the permeability, as might be expected by the minimal morphological and biochemical changes seen. However, permeability in all these dogs was always greater than in the controls, irrespective of the presence of gluten in the diet. Such an underlying permeability defect may be of great importance in the pathogenesis of the condition by allowing access of gluten, or toxic peptides derived from gluten, across the mucosa. In coeliac disease, a persistent defect in the permeability to ${ }^{51} \mathrm{Cr}$-EDTA has been documented in patients in remission on a gluten free diet. ${ }^{33}$

To summarise the results, the dogs reared exclusively on a cereal free diet seem to be more resistant to the damaging effects of gluten on the intestine than those fed a normal diet. Morphological and biochemical changes in biopsy specimens from dogs in the cereal free diet group did occur with gluten challenge but were relatively less severe than in littermates exposed to gluten from weaning. There was no increase in intesti- nal permeability in the cereal free diet group after gluten challenge, probably because there was minimal intestinal damage. However, an underlying permeability abnormality seems to exist, ${ }^{20}$ and this may have allowed the entry of gluten antigens and resulted in the intraepithelial lymphocyte infiltration seen in these dogs after gluten challenge. Although high permeability may induce an increase in intraepithelial lymphocyte density in all susceptible setters in response to gluten, it does not necessarily result in immediate intestinal damage. It has been stated that there is no direct evidence that intraepithelial lymphocyte are directly responsible for the pathogenesis of coeliac disease, ${ }^{34}$ and indeed their role could actually be protective. In untreated coeliac disease, their increased number relative to the numbers of enterocytes largely represent an increase in the density of suppressor $\mathrm{T}$ cells, ${ }^{35}$ and the absolute number of lymphocytes infiltrating the epithelium does not correlate with the degree of intestinal damage as measured by the permeability to ${ }^{51} \mathrm{Cr}$-EDTA. ${ }^{33}$

The ability of gluten to produce appreciable intestinal damage in Irish setters in this study seems to be related to previous exposure to gluten at an early age; early exposure to gluten permits the expression of intestinal damage, and lack of early exposure apparently reduces the damaging effect of gluten. This finding parallels the hypothesis that the expression of coeliac disease is influenced by the age of first exposure to gluten. The appearance of coeliac disease in infancy has been associated with relatively early introduction of cereals into the diet. ${ }^{36}$ Conversely, an apparent drop in the incidence of the disease has been associated with delayed introduction of gluten into the diet of infants because of factors such as prolonged breastfeeding ${ }^{37}$ and the removal of gluten from some commonly used baby foods..$^{6-8}$ However, it is not certain whether the development of coeliac disease in susceptible children has actually been prevented by these changes in feeding practice,${ }^{6}$ as a recent report suggests that introduction of gluten at a later age merely postpones the onset of the disease. ${ }^{38}$ The initial appearance of wheat sensitive enteropathy in dogs reared on a wheat containing diet is slow, ${ }^{18} 19$ and unfortunately it is not clear from the present study whether the lack of response in the cereal free diet group after gluten challenge for six weeks represents true resistance to the disease, or whether the disease would have eventually developed if the challenge had been prolonged. Nevertheless the present study clearly shows that previous exposure to wheat accelerates the recurrence of the disease on subsequent gluten challenge, although questions about the absolute time course of the response to gluten challenge and whether the dose of gluten affects the rate of development remain unanswered. In addition, further studies of the cereal free diet group are needed to determine whether prolonging the duration of gluten challenge or increasing the dose of gluten would have ultimately resulted in full expression of the disease in that group as well.

It seems that the influence of diet in early life may be crucial for the subsequent development of certain intestinal diseases. It has been shown, 
for example, that natural milk feeding in neonatal human infants and guinea pigs promoted a decrease in intestinal permeability, whereas feeding cow's milk formula caused persistently higher intestinal permeability. ${ }^{39}$ Although no morphological changes were present, potentially toxic antigens might have greater access to the mucosa at a critical time in the development of the immune system. It is possible that exposure to gluten earlier in life may be critical for sensitisation of susceptible Irish setters if subsequent gluten challenge is to result in appreciable intestinal damage.

The hypothesis that sensitisation to gluten is an age related event has important consequences. It argues against a biochemical defect being the sole cause of gluten sensitivity in Irish setters, since such a defect would be common to both the diet groups and, therefore, both should remain equally sensitive to gluten. It would also explain why the increased permeability in the cereal free diet group did not result in noticeable intestinal damage after gluten challenge at 1 year of age, even though there was some form of immune response, as indicated by the increase in intraepithelial lymphocyte density. Although intraepithelial lymphocyte infiltration was stimulated by gluten in all susceptible dogs, it is likely that another arm of the immune system is involved in the production of intestinal damage. High intestinal permeability in early life, allowing access of gluten or gluten peptide across the mucosa, could result in damage in susceptible dogs if there was an abnormal immune response; alternatively, access of a potentially toxic gluten fragment in susceptible dogs at a critical time in the development of the immune system, perhaps in association with a biochemical defect in the digestion of gluten, could result in a damaging response, despite a normal immune system. Supported by the Wellcome Trust. Presented in abstract form
at the American Gastroenterology Association Meeting, New at the American
Orleans, May 1988 .

1 Losowsky MS. Coeliac disease. Curr Opin Gastroenterol 1985 1: $235-43$

2 Peters TJ, Bjarnason I. Coeliac syndrome: biochemical mechanisms and the missing peptidase hypothesis revisited. Gut 1984; 25: 913-8.

3 Mearin ML, Biemond I, Peña AS, et al. HLA-DR phenotypes in Spanish coeliac children: their contribution to the underin Spanish coeliac children: their contribution to the under-

4 Walker-Smith JA. Discordance for childhood coeliac disease in monozygotic twins. Gut 1973; 14: 374-5.

5 Polanco I, Mearin ML, Larrauri J, Biemond I, WipkinkBakker A, Peña AS. Effect of gluten supplementation in healthy siblings of children with celiac disease. Gastroenterology 1987; 92: 678-81

6 Dossetor FJB, Gibson AMM, McNeish AS. Childhood coeliac disease is disappearing. Lancet 1981; i: 322-33.

7 Langman MJS, McConnell TH, Spiegelhalter DJ, McConnell RB. Changing patterns of coeliac disease frequency: an analysis of coeliac society membership records. Gut 1985; 26: $175-8$.

8 Stevens FM, Egan-Mitchell B, Cryan E, McCarthy CF, McNicholl B. Decreasing incidence of coeliac disease. Arch Dis Child 1987; 62: 65-8.

9 Bloch R, Menge H, Martini GA, Riecken EO. Effect of gluten on glucose absorption from the small intestine in experion glucose absorption from the small intestine in experi-
mentally induced malabsorption in rats. Digestion 1972; 7: $139-46$

10 Sandhu JS, Fraser DR. Effect of dietary cereals on intestinal permeability in experimental enteropathy in rats. Gut 1983 24: 825-30.
11 Köttgen E, Beiswenger M, James LF, Bauer Ch. In vivo induction of gliadin-mediated enterocyte damage in rats by the mannosidase inhibitor, swainsonine: a possible animal model for celiac disease. Gastroenterology 1988; 95: 100-6.

12 Mowat AM, Ferguson A. Hypersensitivity reactions in the . meaction in the small intestinal mucosa of the mouse. Transplantation 1981; 32: 238-43.

13 Strombeck DR. Small animal gastroenterology. Davis, California: Stonegate, 1979: 234-9.

14 Wagner JD, Jerome CP, Adams MR. Gluten-sensitive enteropathy in a cynomolgus monkey. Lab Anim Sci 1988; 38: $592-4$.

15 Batt RM, Carter MW, McLean L. Morphological and biochemical studies of a naturally occurring enteropathy in the Irish setter dog: a comparison with coeliac disease in man. Res Vet Sci 1984; 37: 339-46.

16 Batt RM, Carter MW, McLean L. Wheat-sensitive enteropathy in Irish setter dogs: possible age-related brush border pathy in Irish setter dogs: possible age-relate

17 Batt RM, McLean L, Carter MW. Sequential morphologic and biochemical studies of naturally occurring wheatsensitive enteropathy in Irish setter dogs. Dig Dis Sci 1987 32: 184-94.

18 Hall EJ, Batt RM. Development of wheat-sensitive enteropathy in Irish setters: morphologic changes. Am $\mathcal{F}$ Vet Res 1990; 51: 978-82.

19 Hall EJ, Batt RM. Development of wheat-sensitive enteropathy in Irish setters: biochemical changes. Am $\mathcal{f}$ Vet Res 1990; 51: 983-9.

20 Hall EJ, Batt RM. Abnormal permeability precedes the development of a gluten sensitive enteropathy in Irish setter dogs. Gut 1991; 32: 749-53.

21 Batt RM, Peters TJ. Subcellular fractionation studies on peroral jejunal biopsies from the dog. Res Vet Sci 1978; 25: 94-100.

22 Hall EJ, Batt RM, Brown A. Assessment of canine intestinal permeability, using ${ }^{\text {s1 }}$ chromium-labeled ethylenediaminetra-acetate. Am F Vet Res 1989; 50: 2069-74

23 McNeish AS, Harms HK, Rey J, Shmerling DH, Visakorp JK, Walker-Smith JA. The diagnosis of coeliac disease. A commentary on the current practices of members of the European Society for Paediatric Gastroenterology and nutrition (ESPGAN). Arch Dis Child 1979; 54: 783-6.

24 Egan-Mitchell B, Fottrell PF, McNicholl B. Prolonged gluten tolerance in treated coeliac disease. In: McNicholl B, McCarthy CF, Fottrell PF, eds. Perspectives in coeliac disease. Lancaster: MTP Press, 1978: 251-7.

25 Peters TJ, Jones PE, Wells G. Analytical subcellular fractionation of jejunal biopsy specimens: enzyme activities, organelle pathology and response to gluten withdrawal in patients with coeliac disease. Clin Sci 1978; 55: 285-92.

26 Hamilton I, Cobden I, Rothwell J, Axon ATR. Intestina permeability in coeliac disease. The response to gluten withdrawal and single-dose gluten challenge. Gut 1982; 23 202-10.

27 Nusslé D, Bozic C, Cox J, et al. Non-coeliac gluten intolerance in infancy. In: McNicholl B, McCarthy CF, Fottrell PF, in infancy. In: McNicholl B, McCarthy CF, Fottrell PF, 1978: $277-86$.

28 Walker-Smith JA, Phillips AD, Rossiter M, Wharton BA. Transient gluten intolerance. Gut 1984; 25: Al190-1.

29 Cooke WT, Holmes GKT. Coeliac disease. Edinburgh: Churchill Livingstone, 1984.

30 Lancaster-Smith M, Packer S, Kumar PJ, Harries JT. Cellular infiltrate of the jejunum after re-introduction of dietary gluten in children with treated coeliac disease. $\mathcal{F}$ Clin Patho 1976; 29: 587-91.

31 Fairman MJ, Scott BB, Toothill C, Losowsky MS. Jejunal mucosal gamma glutamyl transferase activity in coeliac disease. Gut 1977; 18: 484-7.

32 Cooper BT, Candy DCA, Harries JT, Peters TJ. Subcellular fractionation studies of the intestinal mucosa in congenital sucrase-isomaltase deficiency. Clin Sci 1979; 57: 181-5.

33 Bjarnason I, Marsh MN, Price A, Levi AJ, Peters TJ Intestinal permeability in patients with coeliac disease and dermatitis herpetiformis. Gut 1985; 26: 1214-9.

34 Marsh MN. Coeliac disease. In: Marsh MN, ed. Immunopathology of the small intestine. Chichester: John Wiley, 1987: 371-99.

35 Freedman AR, MaCartney JC, Nelufer JM, Ciclitira PJ. Timing of infiltration of $\mathrm{T}$ lymphocytes induced by gluten 40: 741-5.

36 McNeish AS, Anderson CM. The disorder in childhood. In: Cooke WT, Asquith P, eds. Clinics in gastroenterology. Vol 3.
No 1. Coeliac disease. London: WB Saunders, 1974: 127-44.

37 Auricchio S, Follo D, de Ritis G, Marubini E. Does breast feeding protect against the development of clinical symptoms of coeliac disease in children. $\mathcal{F}$ Pediatr Gastrosymptoms of coeliac disease
enterol Nutr 1983; 2: 428-33.

38 Kelly DA, Phillips AD, Elliott EJ, Dias JA, Walker-Smith JA. Rise and fall of coeliac disease 1960-85. Arch Dis Child 1989; Rise and fall of

39 Weaver LT, Laker MF, Nelson R, Lucas A. Milk feeding and changes in intestinal permeability and morphology in the newborn. F Pediatr Gastroenterol Nutr 1987; 6: 351-8. 